\author{
Marcelo E. Marín, PhD \\ Oscar Podestá, PhD \\ Paula Llambías, PhD \\ Francisca Galdón, PhD \\ Viviana M. Scilingo, PhD \\ Daniel Stamboulian, MD \\ FUNCEI (Fundación Centro \\ de Estudios Infectológicos) \\ Ricardo H. Romero, MD \\ Gustavo D. Lopardo, MD \\ Clínica La Sagrada Familia, \\ Buenos Aires, Argentina
}

\section{Nelfinavir in Expanded Postexposure Prophylaxis Causing Acute Hepatitis With Cholestatic Features: Two Case \\ Reports}

\section{To the Editor:}

Two medical professionals treated with postexposure prophylaxis (PEP) after exposure to human immunodeficiency virus (HIV) and hepatitis $\mathrm{C}$ virus (HCV) developed hepatotoxicity probably related to nelfinavir, one of the three drugs in the regimen. It is unlikely that other infections caused the clinical picture.

The Centers for Disease Control and Prevention (CDC) recommends PEP following percutaneous needlestick injuries. ${ }^{1}$ The expanded regimen of three antiretroviral medications, including a protease inhibitor, is indicated after a high-risk exposure., ${ }^{1,2}$ Because of good oral absorption and low reported side effects, nelfinavir is one of the recommended protease inhibitors. Commonly reported side effects involve mild abdominal cramps and diarrhea, which usually can be relieved by over-the-counter remedies. ${ }^{3}$

We report here the results of a chart review of all patients treated with PEP expanded regimen over a 12-month period (August 1998-July 1999) at the University of Connecticut Health Center. Fifteen occupational high-risk exposures to HIV occurred during this period. Of those 15,13 (87\%) received triple antiretroviral therapy with a protease inhibitor, and 4 of these $13(31 \%)$ received nelfinavir. Two of these 4 tolerated the regimen without problems, and the other 2 developed liver toxicity with cholestatic features.

The first patient was a 54-yearold gastroenterologist with a history of polycystic kidney disease who was injured while performing a liver biopsy on a patient infected with HIV and HCV. This physician was stuck on his right index finger, through a single glove, by the 20-gauge needle while anesthetizing the abdominal wall. There was no visible blood in the syringe. The puncture wound was moderately deep and bled immediately. The source patient, who had previously received zidovudine and lamivudine, had a viral load of $14,300 \mathrm{HIV}$ copies, as well as a positive HCV-RNA by polymerase chain reaction (PCR) and antibody titer. His hepatitis B serology was negative.

The gastroenterologist was assessed as having had a high-risk percutaneous exposure to HIV and $\mathrm{HCV}$; his baseline blood tests were within normal limits. He was started on an expanded regimen with zidovudine, lamivudine, and nelfinavir. On day 15 of the regimen, he developed fatigue, diarrhea, sweats, myalgias, and nausea. On day 16 , his temperature was $103^{\circ} \mathrm{F}$, and he developed chills, right upper quadrant abdominal pain, severe diarrhea (12 episodes/day), and weakness. Physical examination demonstrated right upper quadrant abdominal tenderness, no guarding, no rebound, and hepatomegaly, with a $12-\mathrm{cm}$ total liver span. Cultures from blood and urine were negative for aerobic and anaerobic microorganisms; fecal cultures were negative. All three antiviral medications were stopped for 24 hours, and symptoms promptly improved. Zidovudine and lamivudine were restarted on day 17. Ten days after stopping nelfinavir, the physician was feeling better, his liver was $9 \mathrm{~cm}$ (total span), and the abdomen was nontender to palpation. He completed a total of 28 days with antiviral medications; from day 17 to day 28 , he received only zidovudine and lamivudine. Six weeks from the exposure, his entire laboratory tests, including liver enzymes and HIV and HCV antibodies, were normal. Follow-up evaluations at 3 and 6 months revealed normal laboratory results and negative HIV and HCV antibodies; the 12month evaluation showed negative HIV and HCV PCRs.

The second case was a 32-yearold surgical resident. He was assist- ing with the placement of an arteriovenous shunt to prepare an acquired immunodeficiency syndrome patient in renal failure for hemodialysis. During the procedure, another worker passed a bloody \#15 scalpel blade and superficially lacerated the resident's arm through the gown.

The source patient had a viral load of 500 viral copies. The source was positive for $\mathrm{HCV}$ antibodies and negative for hepatitis B surface antigen. The resident physician was assessed as having had a high-risk exposure. His baseline laboratory tests were within normal range. He was started on zidovudine, lamivudine, and nelfinavir. On day 14 after the exposure, the resident developed fever and chills. On day 15 , his temperature was $104^{\circ} \mathrm{F}$, and he felt ill with myalgias, anorexia, and nausea. All antiretroviral medications were stopped for 12 hours. Physical examination revealed fever, clear lungs, nontender abdomen, and shoddy enlarged inguinal nodes. On day 16 , he remained febrile. After 12 hours without antiretroviral medications, he was rechallenged with zidovudine and lamivudine. On day 17 , the physician developed jaundice and abdominal pain, and hepatomegaly was noted. Ultrasonography of liver revealed nonspecific periportal nodes, nonbiliary dilatation, no focal intrahepatic abnormalities, and a thickened gallbladder wall with no stones. On day 21 , the resident tested negative for HCV and HIV by PCR. He continued on zidovudine and lamivudine therapy until day 28 . On day 34 , the liver enzymes were close to the normal range. Follow-up serology at 45 day's, 90 days, 6 months, and 12 months after exposure repeatedly documented negative HIV and HCV PCRs.

The possibility of nelfinavir hepatotoxicity is suggested in these two cases. Nelfinavir has been viewed as an ideal antiretroviral medication because of minimal side effects, which usually are relieved by overthe-counter medications, as well as good bioavailability, varying from $20 \%$ to $80 \%$ after oral administration. Nelfinavir elimination is mostly through the feces $(87 \%)$, and it does not need dose adjustment for patients with renal failure. ${ }^{4}$ In 1998 , the CDC incorporated nelfinavir into the expanded PEP regimen for high-risk percutaneous needlestick injuries.

In the two cases reported, the 
physicians developed symptoms of fever, chills, and myalgias after treatment with nelfinavir. The possibility of acute hepatitis $\mathrm{C}$ was considered, because, in both of these cases, the healthcare workers were exposed to $\mathrm{HCV}$ along with HIV. However, serology for acute $\mathrm{HCV}$ was negative in repeated qualitative testing for hepatitis C viremia by PCR. Additionally, other infectious hepatitis were in our differential diagnosis, including hepatitis $A$ and $G$, as well as cytomegalovirus (CMV) infection. The resident had a negative CMV titer when he developed jaundice. The clinical presentation, with only mild elevations of liver enzymes and rapid improvement following cessation of nelfinavir therapy, suggested most likely a drug-induced problem. Furthermore, there were increases in alkaline phosphatase, right upper quadrant pain, and hepatomegaly, suggesting a cholestatic process in both physicians. ${ }^{5}$ The symptoms promptly subsided with cessation of all medica- tions and continued to improve when the physicians were rechallenged with only zidovudine and lamivudine. The possibility of nelfinavir-induced hepatotoxicity was suspected because of earlier reports of liver toxicity associated with other protease inhibitors. Although there is one case report of acute pancreatitis with nelfinavir therapy, in our two cases, amylases were within normal range. The two patients were monitored closely and agreed to continue with zidovudine and lamivudine after cessation of the three-drug regimen and clinical improvement of symptoms. Diarrhea and nausea also resolved with the discontinuation of nelfinavir. Vomiting and slight dehydration improved rapidly in one patient when he stopped taking nelfinavir.

In summary, our data suggests that nelfinavir may provoke cholestatic hepatotoxicity after the second week of therapy. Although other protease inhibitors have been reported to cause liver toxicity, this is the first report of possible toxic hepatitis from nelfinavir therapy.

\section{REFERENCES}

1. Centers for Disease Control and Prevention. CDC Public Health Service guidelines for the management of health care workers exposures to HIV and recommendations for postexposure prophylaxis. MMWR 1998;47(RR7):1-33.

2. Cardo DM, Bell DM. Bloodborne pathogen transmission in health care workers. Infect Dis Clin North Am 1997;11:331-346.

3. Beach JW. Chemotherapeutic agents for HIV infection: mechanism of action, pharmacokinetics, metabolism and adverse reactions. Clin Ther 1998;20:2-25.

4. Pai V, Nahata M. Nelfinavir mesylate: a protease inhibitor. Ann Pharmacother 1999; 33:325-339.

5. Lewis JH, Zimmerman HJ. Drug and chemical-induced cholestasis. Clin Liver Dis 1999;3:433-464.

Marcia Trapé, MD Sandra Barnosky, APN University of Connecticut School of Medicine Farmington, Connecticut

\section{Eradication of MRSA From a Health Center Ward and Nursing Home}

\author{
Gina Pugliese, RN, MS \\ Martin S. Favero, PhD
}

Long-term healthcare facilities have been recognized as reservoirs of multiresistant bacterial strains, especially methicillin-resistant Staphylococcus aureus (MRSA). Efforts to control MRSA in this setting usually have been only partially effective. Kotilainen and colleagues from Turku University Central Hospital, Turku, Finland, in a recent article describe the eradication of epidemic MRSA from a Finnish healthcare center ward and affiliated nursing home.

The methods to control MRSA included (1) contact isolation precautions, (2) screening for asymptomatic carriage, (3) eradication of carriage, and (4) education of staff on hygienic measures. The first six patients with MRSApositive findings were referred without delay to the infectious diseases unit of the adjacent university hospital for eradication treatment. Later, an isolation unit of six rooms was founded in the healthcare center, where the MRSA-colonized patients were nursed as a separate cohort until they, in succession, were referred to the infectious diseases unit for decolonization.

From May 20 through August 17, 1993, the epidemic MRSA strain was isolated from 8 long-term patients on the $40-$ bed ward of the healthcare center, 4 of the 59 residents of the nursing home, and 1 member of the staff. Eradication of car- riage was successful in all except 1 patient with dementia, who was nursed in contact isolation in the healthcare center until his death 21 months later.

The authors concluded that it is possible to eradicate MRSA from a long-term healthcare facility, even after 13 cases, by applying strict control measures. Their experience may be valuable in the future decision-making process for control of new and more challenging multiresistant bacteria.

FROM: Kotilainen P, Routamaa M, Peltonen R, Evesti P, Eerola E, Salmenlinna $\mathrm{S}$, et al. Eradication of methicillin-resistant Staphylococcus aureus from a health center ward and associated nursing home. Arch Intern Med 2001;26;161:859-863. 